\author{
ARTICULO \\ Revista Derecho - Año 3 edicion 5163 - 176
}

Web: http://revistas.unap.edu.pe/rd E-mail.com: revistaderecho@unap.edu.pe ISSN 2313-6944

\title{
LA TEORÍA BIOLÓGICA COMO CRITERIO RACIONAL EN EL DELITO DE INCUMPLIMIENTO DE OBLIGACIÓN ALIMENTARIA EN EL CASO: 022-2017-INPE/211-83-CTP
}

\author{
Frank Cristian Cabrera Trujillano \\ Juan Vlaney Chambi Acero \\ Rosmery Rivera Illacutipa \\ Rosario Yaneth Tamayo Gurmán
}

INFORMACION DEL ARTICULO

Art. Recibido: 18/03/19 Art. Aceptado: 25/03/19 Art. Publicado: 15/04/19

PALABRAS CLAVE: Conducta criminal Criminología Biotipología Fisionomía criminal Asesor: Abg. Michael Espinoza Coila

\section{RESUMEN}

La criminología a lo largo del tiempo ha ido analizando y desarrollando diversas teorías que intentan explicar el origen de la conducta criminal, dentro de esta se desarrolla la escuela biológica que intenta explicar que la causa se encontraría en alteraciones biológicas, por ende, a nuestra presente investigación queremos demostrar que aún preexiste la aplicación de esta teoría en nuestro país. Dicha postura con el tiempo ha venido perdiendo relevancia, y en el presente en algunos países esta ya no es utilizada o como en el caso peruano esta subrogada a través de otra escuela doctrinaria como lo es la teoría psicológica. 


\section{THE BIOLOGICAL THEORY AS A RATIONAL CRITERION IN THE CRIME OF NON-COMPLIANCE OF FOOD OBLIGATION IN THE CASE: \\ 022-2017-INPE/211-83-CTP}

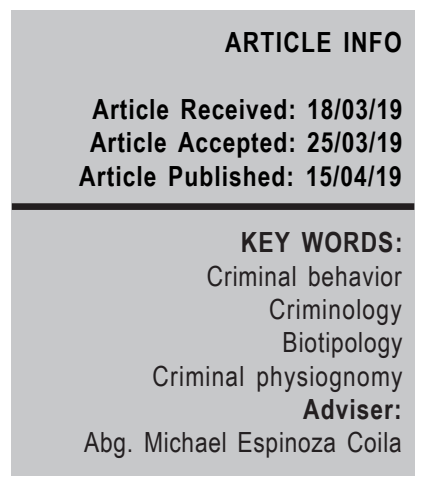

\section{ABSTRACT}

Criminology over time has been analyzing and developing various theories that try to explain the origin of criminal behavior, within this develops the biological school that tries to explain that the cause would be in biological alterations, therefore, to our present research we want to show that the application of this theory in our country still pre-exists. This position with time has been losing relevance, and in the present in some countries this is no longer used or as in the Peruvian case is subrogated through another doctrinal school as is psychological theory.

164 Revista Derecho-5 (2019) 


\section{INTRODUCCION}

En el presente trabajo pretendemos es analizar la teoría biológica relacionado con el caso designado que trata de «Incumplimiento de Obligación Alimentaria» Con la finalidad de relacionar entre el lado físico y el lado moral del individuo se realizaron numerosas investigaciones con el convencimiento de que los trazos fisonómicos servían para reconocer los caracteres psíquicos sobresalientes de las personas; es decir, el modo de pensar y de sentir de cada individuo. Fruto de estas investigaciones surgió la denominada fisionomística, o cuyos principales exponentes fueron Aristóteles, Sócrates, Galeno, entre otros; esta disciplina tuvo su apogeo en la Edad media a través de las denominadas ciencias ocultas de la quiromancia, podomancia, y otras; las cuales pretendían reconocer el carácter de las personas por las líneas de las manos, de los pies y hasta por el ombligo.

Otro gran investigador acerca de esta teoría es Lombroso su teoría es más de carácter referencial e histórico, porque marcó un hito precursor de la Criminología; pero hoy en día no es posible pensar en la existencia de un delincuente natural o «nato», por cuanto el comportamiento humano se explica por una interrelación de factores el intento de buscar un «tipo criminal» basándose en los estudios constitucionalistas y biotipológicos fue importante para la criminología como estudio del hombre y la criminalidad, pero no fue trascendental, puesto que los delincuentes no pueden conformar un «tipo» que no sea el de los que no han delinquido, aunque estudios como los de Kretschmer, Sheldon y otros, admitan la evidencia de que los tipos anatómicos, con determinada morfología, influyen en los caracteres somáticos propios, formándose tipos psíquicos y somato-psíquicos, como veremos más adelante.

\subsection{LA BIOTIPOLOGÍA CRIMINAL}

La biotipología criminal es la ciencia del tipo humano, el cual es concebido como una unidad vital (biotipo), con varias facetas: Morfología, fisiología y psicología; esta es una disciplina científica cuyas precursoras fueron la fisonomía y la psicología; versa sobre el tipo humano atendiendo al predominio de un órgano o función; su premisa es que existe una correlación entre las características físicas del individuo y sus rasgos psicológicos, entre el tipo somático o corporal y el tipo mental o temperamento. La biotipología busca establecer correlaciones entre las formas corporales y el temperamento, considerando que a determinada constitución somática corresponden ciertos rasgos temperamentales y conductuales.

Revista Derecho - 5 (2019) 165 
La Biotipología en palabras de Rodríguez Manzanera (1981) ha seguido un largo camino, que básicamente muestran la búsqueda de las relaciones entre las características físicas de un individuo y sus características psicológicas. Así, los primeros estudiosos fueron los Fisionomistas, para luego continuar con los Frenólogos, por ello se considera a estos dos como los antecesores de la moderna Biotipología.

«La Biotipología Criminológica sería la aplicación de los conocimientos biotipológicos para la distinción de diversos tipos entre los criminales».(Manzanera, 1981)

\subsubsection{ESCUELAS BIOTIPOLÓGICAS}

\subsection{LA ESCUELA FRANCESA.}

En la Escuela francesa destaca Claudio Sigaud (1862-1921), desarrolla la llamada «morfología» del delincuente, en la cual los distinguía según su forma exterior como: planos y redondos; dando dos tipos primarios: retraidos y dilatados (Manzanera, 1981).

La teoría de Sigaud produce entonces cuatro «tipos» de humanos según el sistema que predomine en los mismos (respiratorio, digestivo, muscular y cerebral), sistemas que conectan a su vez, con los cuatro medios principales (atmosférico, alimenticio, físico y social). Cada «tipo» según Sigaud, tiene sus propias características: El tipo respiratorio presenta tórax, cuello y nariz largos, senos de la cara desarrollados y particular sensibilidad a los olores; el tipo digestivo presenta boca y maxilar inferior grandes, ojos chicos y cuello corto, tórax ancho y abdomen desarrollados, propios de individuos obesos; el tipo muscular presenta desarrollo armónico de esqueleto y músculos así como los tres pisos faciales; y el tipo cerebral, figura frágil y delicada, frente grande y extremidades cortas. (Verela, 2017)

\subsection{LA ESCUELA ITALIANA}

Jacinto Viola señala que la constitución humana descansa en dos sistemas: el sistema de la vida vegetativa o visceral y el de la vida de relación o nervioso y muscular, de donde se extraen dos tipos: el brevilíneo y el longilíneo.(Villanueva, 2001)

En el tipo brevilíneo, el desarrollo del cuerpo es horizontal, el predominio del sistema vegetativo produce individuos enérgicos, alegres y vitales. El tipo longilíneo predomina la altura, tórax alargado, abdomen plano y miembros largos, abúlicos y depresivos, con tendencia a la introversión y a la fantasía, representa la vida de relación.(Manzanera, 1981) 
Nicola Pende elabora su tipología considerando, también, factores endocrinológicos Verela (2017); y clasifica a los seres humanos en: longilíneoesténico (individuos fuertes, delgados, con hiperfunción de tiroides y suprarrenales), el longilíneo-asténico (débiles, delgados, de escaso desarrollo muscular e hipofunción de las suprarrenales), el brevilíneo-esténico (fuertes, musculados, de reacciones lentas, de baja estatura, con hipotiroidismo y con hiperfunción de las suprarrenales) y el brevilíneo-asténico (gordos, débiles, lentos de reacciones y con hipofunción de pituitaria y tiroides) (Manzanera, 1981).

\subsection{LA ESCUELA ALEMANA}

Ernest Kretschmer (1888-1964), es el máximo representante de esta escuela, en su obra se propuso hallar las correlaciones entre la estructura somática y la psíquica, es decir, comprobar si a determinados tipos somáticos corresponden determinados tipos psíquicos y viceversa (Manzanera, 1981).

Kreschner elabora una doble clasificación tipológica, distingue, por una parte, los tipos leptosomático: cuerpo alargado y delgado, cabeza pequeña, nariz puntiaguda (su representación gráfica es una línea vertical); el tipo atlético: gran desarrollo del esqueleto y musculatura, tórax y cabeza grande (su re- presentación geométrica es una pirámide invertida); el tipo pícnico: Gran desarrollo de las cavidades viscerales, abdomen prominente, cabeza redonda y ancha, extremidades cortas y tendencia a la obesidad (representación circular); y el tipo displástico: Que cuenta con características muy exageradas y son individuos que no encajan en los tipos anteriores, con tres variantes o subtipos (como el gigantismo, la obesidad o el infantilismo eunocoide) (Verela, 2017)

Relacionando los tipos constitucionales anteriores y reduciendo las características psicológicas, Kretschmer formula una segunda tipología: tipos esquizotómicos(introvertidos, leptosómicos), ciclotómicos (extrovertido, pícnico) y viscosos(atléticos, pasivos, tranquilos). (Verela, 2017)

En cuanto a las relaciones entre biotipo y criminalidad, Kretschmer llega a la conclusión de que los pícnicos arrojan los índices más bajos de criminalidad, constituyéndose raras veces en delincuentes habituales; los leptosomáticos son de difícil tratamiento y proclives a la reincidencia, siguiendo a los atléticos en porcentajes de criminalidad; abundan entre estos los ladrones y estafadores; los atléticos son violentos y representan los índices más altos de delincuencia.

Revista Derecho - 5 (2019) 167 
1.5. LA ESCUELA AMERICANA.

La clasificación más usada en Norteamérica es la creada por William Sheldon y S.S. Stevens; quienes tienen una clara ventaja por partir de un plano experimental.

Partiendo del blastodermo que es la célula de la cual provenimos todos, reconoce tres dimensiones: el endodermo (las vísceras digestivas huesos, músculos, tendones, etc.), el mesodermo (del sistema motor), y el ectodermo (el tejido nervioso, piel, etc.)

Llevan una escala de puntos, por la cual (1) es el sujeto que carece de determinado rasgo y (7) lo tiene claramente determinado, Sheldon manejó un total de 60 rasgos (postura, sociabilidad, apetito, afectividad, tolerancia, ambición, aventura, etc.). (Manzanera, 1981).Las características físicas o componentes estáticas como las llama Sheldon, citado por Manzanera (1981), son:

- Endomorfo; evidenciaría: vísceras digestivas pesadas y muy desarrolladas, con estructura somática relativamente débil; bajo peso específico, tendencia a la gordura, formas redondeadas, miembros cortos, piel con vello y suave.

- Mesomorfo; tendría un gran desarrollo de las estructuras somáticas (huesos, músculo, tejido conjuntivo), alto peso específico, dureza, erecto, fuerte, resistente, tronco grande, pecho consistente, de manos grandes.

Ectomorfo; presentaría un cuerpo frágil, alargado, delicado, con extremidades largas y delgadas, músculos pobres, tórax chato, huesos poco consistentes y finos, hombros caídos cara pequeña, nariz, afilada y pelo fino.

A cada tipo físico o corporal le corresponderían unos rasgos caracterológicos y temperamentales propios de tres tipos respectivamente: el tipo viscerotónico, el somatotónico y el cerebrotónico (Verela, 2017).

El tipo viscerotómico es endomorfo, cómodo, lento, glotón, sociable, cortés, amable, tolerante, hogareño, extrovertido.

El somatotónico es mesomorfo, firme, aventurero, energético, atlético, ambicioso, osado, valiente, agresivo, inestable, escrupuloso, estridente, dinámico.

El cerebrotónico es ectomorfo, rígido, rápido, aprensivo, controlado, asocial, desordenado, hipersensible, solitario, pleno de problemas de carácter funcional, alergias, insomnios, sensible al ruido, introvertido, etc.

Luego de las pruebas experimentales, Sheldon concluye que al aplicarse

168 Revista Derecho - 5 (2019) 
la clasificación a grupos de delincuentes, se encuentra que la mayoría son mesomorfos, teniendo la variante de $60.1 \%$ (delincuentes) contra un 30.7\% (no delincuentes) (Manzanera, 1981).

\section{LAS FAMILIAS CRIMINALES}

Durante la evolución de la criminología, varios orientaron sus estudios a las familias criminales, descomponiendo de ella una verdadera genealogía criminal, encontrando concordancias notables, y demostrando que existen familias célebres en las que la mayoría de los componentes son criminales.

Como el profesor (Rodriguez Manzanera, 1981) Tenemos un ejemplo clásico es la familia Juke, seguida durante 200 años por Dugdale, en que se probó que el fundador de esta familia, un malviviente alcohólico, tuvo 709 descendientes, de los cuales 77 delincuentes, 202 prostitutas y 142 vagos y malvivientes. Estabrook amplió las investigaciones, hasta localizar 3,000 descendientes, de los cuales la mitad son deficientes mentales, y un tercio vagos, mendigos, prostitutas y delincuentes. Hurwitz hace ver cómo el número de delincuentes va decreciendo con el tiempo.

Sin embargo, varios especialistas en lo sociológico sostienen que esto era producido por el aprendizaje derivado de la convivencia, pues si los padres eran criminales, los hijos viendo ello se inclinaban por dicha conducta.

La crítica más generalizada para estas investigaciones es que la obtención de métodos no es totalmente confiable, pues el diagnóstico de debilidad mental, o la calificación de vagos o prostitutas se hace con base en referencias generalmente verbales, y en ocasiones con un siglo de diferencia.

\subsection{LA ESTADÍSTICA FAMILIAR}

Para vencer las dificultades anteriores, varios investigadores se preocuparon por usar la estadística y buscar datos más directos, así como formas de comparación o control.

Goring y Lund citado por (Rodriguez Manzanera, 1981) coinciden en sus estudios al encontrar que la proporción de delincuentes condenados a prisión (por delitos graves) es mayor entre aquellos en los que ambos padres fueron delincuentes, que entre aquellos en los que un solo padre fue condenado, y estos últimos son más que aquellos sin padres con antecedentes criminales.

O también el estudio planteado por Bernhardt citado Rudolf en 1930, quien efectuó un estudio sobre crimina-

Revista Derecho - 5 (2019) 169 
les dividiendo en dos grupos: a) Aquellos cuyos padres no era criminales, pero los abuelos y otros ascendientes sí. b) Aquellos sin parientes criminales. El resultado es que en el grupo «a» la proporción de hermanos delincuentes es el doble que en el grupo «b», a pesar de que ambos ambientes fueron considerados «no criminógenos».

Un grupo bastante apreciable de investigadores se dedicó a buscar taras hereditarias de delincuentes convictos, distinguiendo taras, directas (padre-madre), y taras en general (ascendientes).

\section{LAS ABERRACIONES} CROMOSOMATICAS

\subsection{ABERRACIONES CROMOSOMÁTICAS Y CRIMINALIDAD}

El descubrimiento de un alto número de prisioneros con aberraciones cromosomáticas (en relación a la población general), llevó a estudios sobre las relaciones entre aberración y criminalidad. Las aberraciones cromosomáticas en las mujeres no habían demostrado ser significativas en relación a la delincuencia, planteándose la hipótesis de ser ésta una de las causas por las que la delincuencia femenina es proporcionalmente menor a la masculina.

El profesor (Rodriguez Manzanera, 1981) hace la siguiente com- pilación, cuyos datos son acorde a diversos autores que, tras un arduo estudio, merecen el mérito de ser mencionados; sin embargo, se ha podido demostrar que el $0.16 \%$ de las mujeres tienen aberración gonosómica (más de una x), en tanto que en las cárceles de máxima seguridad el número se eleva a $0.4 \%$. Este descubrimiento pudo confirmarse en el estudio de la cárcel de mujeres de la ciudad de méxico, pues se pudo detectar un $0.45 \%$ (una $\mathrm{xxx}$ sobre 221 reclusas). En sujetos con síndrome de klinefelter (xxy, xxxy, xxxxy), se ha encontrado $2.5 \%$ de delincuentes débiles mentales. En las poblaciones penitenciarias se ha encontrado $2.4 \%$ de delincuentes con factor xyy, mientras en la población no delincuente la relación es de 2.3 por lo anterior representaría que entre los delincuentes encontramos mayor número de xyy que entre la población normal. el fenómeno puede comprenderse, ya que los sujetos con xyy presentan características de peligrosidad como son: precocidad criminal, «yo» mal estructurado, mala adaptación social, poca tolerancia a la frustración, mayor reincidencia, falta de sentido de responsabilidad, incapacidad de previsión, etc. Son sujetos de gran precocidad criminal (13.1 años para la comisión del. primer delito, frente a 18 años de la generalidad). en general son buenos reos, se adaptan rápido al establecimiento, no dan muchos problemas, pero son refractarios al tratamiento y reinciden con gran facilidad. en el grupo de control obser-

170 Revista Derecho - 5 (2019) 
vado se encontraron que había mayor número de problemas dentro de las instituciones penitenciarias. esto sin duda va a confirmar aquel conocimiento expuesto por los grandes maestros de criminología, de que los peores delincuentes son los mejores presos.

Entre los sujetos con factor xyy detectados, el 5 1\% había tenido serios problemas de comportamiento. el 70\% de los sujetos con factor $\mathrm{xx}$ y yy que registra la literatura médica tuvieron problemas de conducta.

El Dr. Quentin de Bray ha revelado que hay algunas otras anomalías aparte de la XXY y de la XYY, como aquella de un solo cromosoma $\mathrm{Y}$, pero de un tamaño insólito, el cual puede estar asociado al fenómeno criminal.

Los sujetos con un cromosoma $\mathrm{Y}$ gigante desarrollan mayor estatura y son más agresivos que aquellos que tienen el cromosoma $Y$ de tamaño normal. Existe ya una abundante casuística para ilustrar el tema, y los estudios se han multiplicado.

En lo que a México concierne los resultados han sido los siguientes: En su tesis recepcional (1970) el señor Alfonso González Noriega, de la Facultad de Ciencias de la UNAM, hace un detenido estudio de la cromatina sexual en la población de pacientes de dos hospitales psiquiátricos de México, encon- trando una incidencia de cromatina sexual anormal, más alta en pacientes de hospitales psiquiátricos que en recién nacidos, concluyendo que parece razonable asumir que un cromosoma «X» adicional juega un papel significante en la inducción de enfermedades mentales.

En otro estudio sobre enfermos mentales en hospitales mexicanos, se encontró: 3 Klinefelter (Uno XXXXY) en 300 niños; 1 Klinefelter en 300 adultos hombres; y 1 Turner en 377 mujeres." En 2 penitenciarías para hombres se localizaron: 2 Klinefelter (XXY) en 78 reclusos; y $1 \mathrm{XXY}$ en 236 internos, o sea 3 XXY en 314 delincuentes $(0.95 \%)$.

Aunque se encontraron 2 mosaicos XYY (aberración en algunas células $\mathrm{y}$ en otras no), no se ha localizado un solo caso de XYY entre delincuentes mexicanos. La explicación de esta ausencia está aún en discusión, aunque pudiera interpretarse como muestra muy pequeña o por la negativa de parte de la población de someterse al examen.

\subsection{CROMOSOMAS Y CRIMINALIDAD Y CONSIDERACIONES}

El exceso de gonosomas puede originar una predisposición a los trastornos de conducta, pues el sujeto afectado posee un terreno especial que lo hace más sensible que el resto de las perso-

Revista Derecho - 5 (2019) 171 
nas, ante estímulos criminógenos ambientales.

Parece existir una correlación positiva de la existencia de una doble YY a la agresividad, siendo ésta una característica más masculina que femenina.

Aunque el número de delincuentes con este problema es muy bajo $(2.5 \%$ para Klinefelter y $2.8 \%$ para XYY), es indudable que los estudios de genética nos han dado nuevos y valiosos elementos para la comprensión del fenómeno antisocial.

\section{ANÁLISIS DEL CASO DE INCUMPLIMIENTO DE OBLIGACIÓN ALIMENTARIA EXPEDIENTE JUDICIAL N'00077-2016-92-2101-JR-PE-01}

El expediente empieza con la solicitud de beneficio penitenciario de liberación condicional para los cual se presentarán diferentes datos y documentos que nos ayudarán para el análisis criminológico de la teoría biológica.

\section{* Datos del interno:}

El presente expediente empieza con la ficha de información general, el interno se llama C. H. E. T.; el lugar de nacimiento del interno es Azángaro de nacionalidad peruana su fecha de nacimiento es el 24 de Julio de 1961 tiene un nivel de instrucción 5to de secunda- ria de estado civil conviviente con 7 hijos, pero 1 hijo falleció.

El delito por el cual está recluido es la omisión a la asistencia familiar, el tiempo de condena es de 4 meses y es su primer ingreso a un centro penitenciario, la fecha de ingreso al establecimiento penitenciario es el 16 de diciembre del 2016.

\section{* Hechos imputados por el Ministerio Publico:}

Ante el tercer juzgado de Puno se ha tramitado el expediente de alimentos $\mathrm{N}^{\circ} 1719-2012$, habiéndose dispuesto el pago de una pensión de alimentos en favor del agraviado en la suma de 280.00 soles ante su incumplimiento se generaron requerimientos de pago y devengados en la suma de S/. 7503.86 además se han fijado por concepto de reparación civil el monto de $\mathrm{S} / .250 .00$ que hacen un total de S/. 1503.86.00

\section{* Posición de la parte acusa:}

El acusado admitió tener responsabilidad penal, y también admitió sentirse responsable por los daños ocasionados a la parte agraviada, y solicita someterse a la conclusión anticipada, teniendo en cuenta que no tiene antecedentes penales ni policiales, también el presenta un certificado de conducta donde indica que según la conducta que obran en el colegio técnico penitencia-

172 Revista Derecho - 5 (2019) 
rio de este establecimiento penitenciario no registra sanción disciplinaria, también presenta un certificado de domicilio, presenta un contrato de trabajo a plazo fijo bajo la modalidad de contrato de temporada.

\section{* Informe psicológico:}

Tenemos que tener en cuenta una parte importante del examen psicológico donde se menciona las particularidades físicas, en la cual indica que el interno se caracteriza por ser de raza mestiza, de aproximadamente $1.61 \mathrm{~cm}$ de altura y contextura pesando los 64 kilos, de presencia arreglada y aseo adecuado, aparenta la edad cronológica mencionada, de caminar pausado y postura erguida, no se observan cicatrices ni tatuajes a simple vista, pero existe un lunar en el lado derecho del rostro.

El interno refiere que conoció a su primera pareja cuando él tenía 19 años de edad y ella tenía 21 fruto de esa relación procrearon 6 hijos, pero uno falleció de neumonía cuando tenía 7 meses de nacido, ellos tuvieron una convivencia de 35 años. El interno tenía 49 años cuando conoce a su segunda pareja quien tiene 29 años trabajaba como dama de compañía en un night club en desaguadero, el interno reside en Arequipa junto a la familia que formo.

\section{v Historia conductual durante la primera etapa segunda infancia y adolescencia.}

Son 9 hijos de padre y madre refiere que nunca conoció a sus padres porque a los 2 días de nacido su papa lo regalo a una familia y esta así ves tenía una hija los padres que lo adoptaron laboraban en la agricultura él se entera que es adoptado a los 10 años de edad sufrió mucho con la noticia los familiares de su padre adoptivo le dan la noticia.

\section{* Comportamiento durante la permanencia en EP}

El interno que se caracteriza por no tener sanciones disciplinarias, durante su vida punitiva desde que ingreso a este establecimiento ha estado inscrito en el área de trabajo de elaboración de monederos, billeteras, carteras en cuero.

\section{* Características psicológicas}

Cuando se le examina las pruebas psicológicas se observan que posee una tipología con tendencia a la estabilidad y a la introversión característica de un temperamento melancólico con funciones psíquicas básicas con respecto a la atención existente persistencia de la concentración. 


\section{* Apreciación psicocriminologica}

EI interno de 55 años de edad de raza mestiza, contextura leptosomica de presencia arreglada y aseo adecuado, aparenta la edad cronológica mencionada, de condiciones socioeconómicas regular refiere el interno, mediante las pruebas psicológicas se observa que posee una personalidad independiente, latamente desconfiado.

\section{CONCLUSIONES}

La teoría biológica como vimos parte de un fundamento racional entendiendo que el cuerpo humano es fuente de información compleja que a su entendimiento se logra identificar cuestiones científicas que influencia el comportamiento de un criminal, en todo caso nos referimos al proceso que se desencadena a partir de una acción conllevado a una reacción; aquellos factores pueden ser las enfermedades corporales, las enfermedades mentales, el ente humano interno (biológico); se basan, en compleja y parte a los procesos biológicos entre los que se distinguen ciertos factores que influyen en el desarrollo anormal de una persona. Esta posición que permaneció fuerte durante más de cinco décadas, posteriormente pasó al psicologismo y a la psiquiatrización al considerar al sujeto criminal como un sujeto trastornado, pasados los años, la nueva corriente fue la del sociologismo, pero en la actualidad, la tendencia es multifactorial, aunque se ha abandonado en mucho la perspectiva de nacimiento de la Criminología. Por lo anterior, es necesario conocer de éstos distintos enfoques para que el Criminólogo se oriente por alguno de éstos o considere a todos para el entendimiento causal de la criminalidad, el caso que aquí ocupa es el de la Genética, la Biología y la Endocrinología, siendo importantes porque solo de esta forma se podrá entender de manera científica la reacción del ser humano al alterar su estado físico-mental así, se podrá implementar políticas que eviten el estrés criminal en la sociedad actual.

Muchas han sido las teorías realizadas a lo largo de la historia que han intentado averiguar el origen y las causas de la delincuencia. Los estudios muestran que no nos podemos quedar con una sola teoría explicativa sino más bien con un compendio de ellas; lo que llevaría a confeccionar un modelo explicativo biopsicosocial de la conducta antisocial, dentro de la interacción individuo-ambiente. Por tal razón cada uno de los acercamientos aquí descritos para explicar la delincuencia se complementan entre sí para tener una imagen más completa del fenómeno de la delincuencia, abordando los múltiples factores involucrados en la génesis de la misma. El caso analizado en el ámbito criminal rosario es la clara evidencia de la inferioridad del ser humano cuyo aspecto

174 Revista Derecho-5 (2019) 
conllevan a determinar la conducta criminal, evaluando los antecedentes, fundamentos y consecuencias, se llega a afirmar que cada situación concreta que conlleva y conduce a realizar determi- nadas acciones se adapta en la biología, la fisionomía, y entre otros aspectos, influyen en la deducción de los especialistas a definir y posteriormente colaborar con la criminología. 
176 Revista Derecho-5 (2019) 